\title{
Infestação por Lynxacarus radovskyi (Tenório, 1974) em gatos (Felis domestica), no Município de Porto Velho-RO
}

\author{
Infestation by Lynxacarus radovskyi (Tenório, 1974) in cats (Felis domestica), in Porto Velho-RO \\ Infestación por Lynxacarus radovskyi (Tenório, 1974) en gatos (Felis domestica), en Porto Velho-RO
}

\author{
Thiago Vaz Lopes \\ ORCID: https://orcid.org/0000-0002-3192-1908 \\ Universidade Federal do Acre, Brasil \\ E-mail: thiagovlopes@hotmail.com \\ João Gustavo da Silva Garcia de Souza \\ ORCID: https://orcid.org/0000-0002-5899-2490 \\ Centro Universitário Aparício Carvalho, Brasil \\ E-mail: joaogarciadesouza27@gmail.com \\ Clívia de Melo Pessôa \\ ORCID: https://orcid.org/0000-0002-5405-873X \\ Centro Universitário Aparício Carvalho, Brasil \\ E-mail: cliviademelo63@gmail.com \\ Paloma Gabrielle Lopes Leão \\ ORCID: https://orcid.org/0000-0002-9657-1910 \\ Centro Universitário Aparício Carvalho, Brasil \\ E-mail: palomaleaomedvet@gmail.com \\ Sandro de Vargas Schons \\ Fernando Andrade Souza \\ ORCID: https://orcid.org/0000-0002-9474-9404 \\ Universidade Federal do Paraná, Brasil \\ E-mail: femedvet@yahoo.com.br
}

\begin{abstract}
Resumo
O Lynxacarus radovskyi, um dos principais ácaros que acometem felinos, é um Sarcoptiforme que pertence à família Listophoridae. Caracteriza-se por ser um importante causador de dermatopatia, onde a maioria dos casos se apresentam na forma subclínica, e as manifestações clínicas são proporcionais à quantidade de ácaros no animal, transmitidos principalmente através de fômites e contato direto; os sinais clínicos rotineiramente observados são alopecia, descamações, pelos facilmente epilados, prurido e dermatite miliar. O presente trabalho objetivou relatar a ocorrência de Lynxacarus radovskyi, seus aspectos sintomatológicos e o tratamento estabelecido, em um grupo de 26 gatos domésticos, provindos de um gatil comercial, na cidade de Porto Velho - RO, visto que não existe outro relato anterior a esse no Estado. O tratamento dos animais foi realizado com banhos de Amitraz e a pulverização de Fipronil Spray®, semanalmente, no prazo de 30 dias, demonstrando ser eficaz para a eliminação do agente, com os animais sadios ao fim do período de tratamento.
\end{abstract}

Palavras chave: Linxacariose; Ectoparasita; Gatos; Parasitologia; Tratamento.

\section{Summary}

Lynxacarus radovskyi, one of the main mites that affect felines, is a Sarcoptiform that belongs to the Listophoridae family. It is characterized for being an important cause of dermatopathy, in which most cases present in the subclinical form, and the clinical manifestations are proportional to the amount of mites in the animal, transmitted mainly through fomites and direct contact; the clinical signs routinely observed are alopecia, scaling, easily piled hair, pruritus, and miliary dermatitis. The present work aimed to report the occurrence of Lynxacarus radovskyi, its symptomatological aspects and the established treatment, in a group of 26 domestic cats, coming from a commercial cattery, in the city of Porto Velho - RO, since there is no other report prior to this one. in the State. The treatment of the animals was carried out with baths of Amitraz and the spraying of Fipronil Spray®, weekly, within 30 days, proving to be effective for the elimination of the agent, with healthy animals at the end of the treatment period.

Keywords: Linxacariosis; Ectoparasite; Cats; Parasitology; Treatment.

\section{Resumen}

Lynxacarus radovskyi, uno de los principales ácaros que afectan a los felinos, es un sarcoptiforme que pertenece a la familia Listophoridae. Se caracteriza por ser una importante causa de dermatopatía, en la que la mayoría de los casos se 
presentan en forma subclínica, y las manifestaciones clínicas son proporcionales a la cantidad de ácaros en el animal, transmitidas principalmente a través de fómites y contacto directo; los signos clínicos que se observan habitualmente son alopecia, descamación, pelo que se amontona fácilmente, prurito y dermatitis miliar. O presente trabalho objetivou relatar a ocorrência de Lynxacarus radovskyi, seus aspectos sintomatológicos e o tratamento estabelecido, em um grupo de 26 gatos domésticos, provindos de um gatil comercial, na cidade de Porto Velho - RO, visto que não existe outro relato anterior a esse en el estado. El tratamiento de los animales se realizó con baños de Amitraz y la aspersión de Fipronil Spray®, semanalmente, dentro de los 30 días, demostrando ser eficaz para la eliminación del agente, con animales sanos al final del período de tratamiento.

Palabras clave: Linxacariosis; Ectoparásito; Gatos; Parasitología; Tratamiento.

\section{Introdução}

Sendo vista como uma doença difícil de se encontrar nos gatos, a linxacariose tem como seu agente etiológico o Lynxacarus radovskyi, um ácaro de formato sarcóptico, proveniente da famila Listrophoridae (Heath \& Mariadas, 1999). Têm cerca de $0,5 \mathrm{~mm}$ e entre 430 e $515 \mu \mathrm{c}$ de comprimento, que normalmente ficam ligados na região do terço distal da haste do pelo, podendo ser observado a olho nu (Scott, Miller \& Griffin, 1996).

Em sua maioria, as infecções por este agente se dão através do contato direto com o mesmo. Porém, os fômites podem adquirir um papel crucial nas transmissões (Craig \& Tell, 1993). Após o animal se contaminar com o parasita, demonstra alopecia, prurido e começa a obter uma aparência de sujeira extrema, visto que se agarram aos pelos, o que gera uma sensação de pelos salpicados, como "sal e a pimenta". Além disso, os pelos dos animais com linxacariose se soltam de forma muito fácil. A derme pode ser encontrada normal ou apresentando erupções papulares, caracterizando uma dermatite miliar (Faustino, Messias \& Romeiro, 2004).

Seu primeiro relato foi realizado por Tenório (1974) no Havaí. Além disso, há relatos somente em alguns países, como os Estados Unidos, Porto Rico, Nova Zelândia, Ilhas Fiji, Austrália e o Brasil (Romeiro et al., 2007; Aguiar et al., 2009), sendo a sua primeira vez relatada no país em 1986 (Facchini \& Coutinho, 1986). Mesmo sendo muito incomum, já foi relatado casos em oito estados diferentes do país, sendo eles Alagoas, Rio de Janeiro, Bahia, Rio Grande do Norte, Pernambuco, Rio Grande do Sul, São Paulo e Pará (Porto et al., 2002; Aguiar et al., 2009).

Apesar de pouco diagnosticada, a linxacariose felina é uma enfermidade dérmica muito frequente em felinos, mas que existe poucos relatos pois muitas vezes pode ser apenas subdiagnosticada. Contudo, é de suma importância que na clínica médica de felinos ela deve ser mais descrita. Por este fato, essa afecção cutânea deve ser mais estudada e relatada, tanto em estudo científicos, como na própria prática clínica (Oliveira \& Vieira Neto, 2019).

Objetivou-se com esse trabalho relatar a ocorrência do Lynxacarus radovskyi, os seus aspectos sintomatológicos e o tratamento estabelecido, em um grupo de 26 gatos domésticos, provindos de um gatil comercial, da cidade de Porto Velho - RO, sendo este o primeiro trabalho relatando a presença da linxacariose no estado de Rondônia.

\section{Relato de Caso}

Foram atendidos vinte e seis felinos da raça Persa, machos e fêmeas de diferentes idades, em um Hospital Veterinário da cidade Porto Velho-RO), provindos de um gatil comercial. Os animais apresentavam pelagem com o aspecto de polvilhado de "sal e pimenta", característico de linxacariose, lesões alopécicas multifocais, prurido generalizado, descamação, dermatite úmida, vômito com grande quantidade de pelos, anorexia e emagrecimento crônico.

Para a obtenção do diagnóstico, foi realizado uma tricograma nos animais suspeitos. Após a coleta de material, utilizouse de lâminas e um microscópio para conseguir efetuar a identificação do ácaro, bem como os seus ovos, na análise microscópica, podendo ser observado nas imagens 1 e 2 a presença de ovos e o agente parasitário adulto, respectivamente. 
Imagem 1. Ovos de L. radovskyi visto por miscrocópio, na objetiva de 100x.

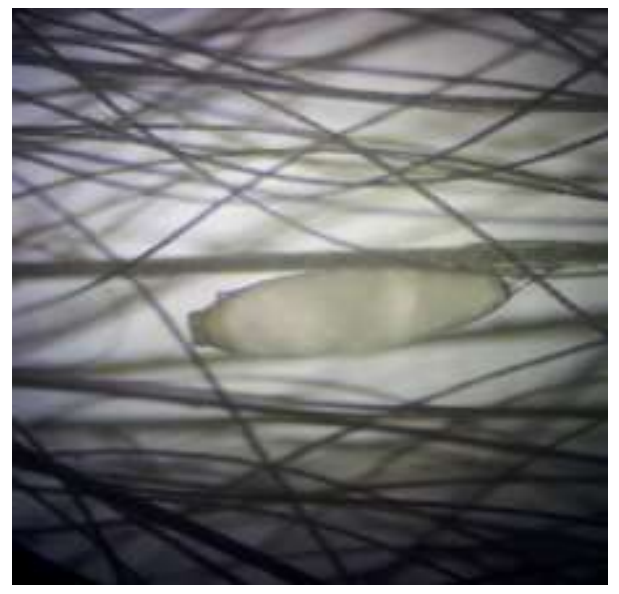

Fonte: Autores.

Imagem 2. Lynxacarus radovskyi adulto em microscópio, na objetiva de 100x.

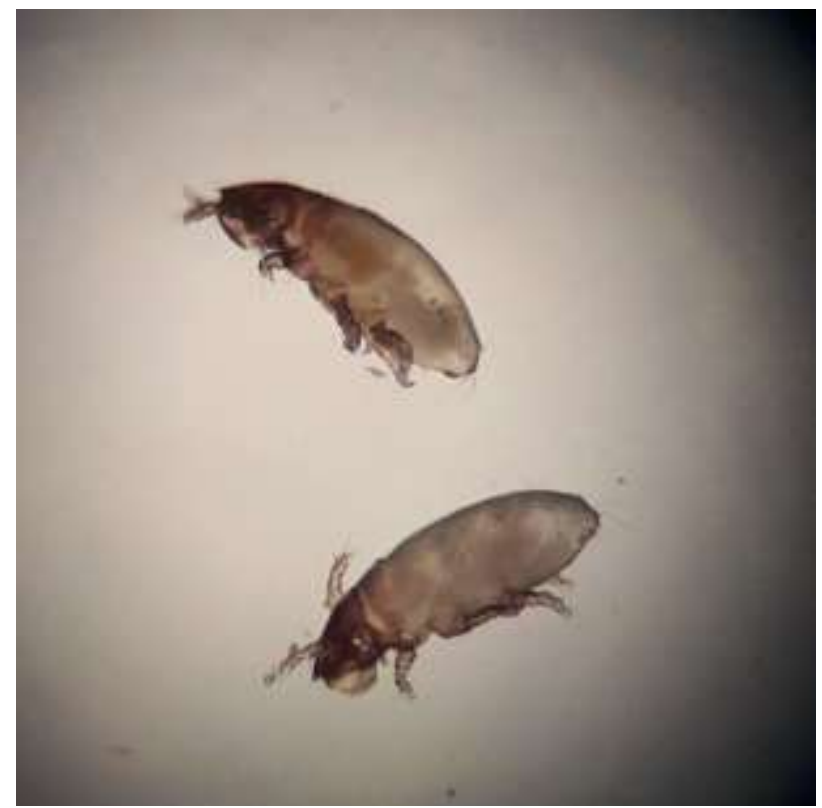

Fonte: Autores.

O tratamento dos animais consistiu na realização de banhos com Amitraz e a pulverização de Fipronil Spray®, semanalmente, durante um período de 30 dias. Além disso, foi realizado a desinfecção das instalações, com aspersão de Amitraz em todo o ambiente. Chegado o prazo para o término do tratamento, foram então efetuados novos testes em todos os animais acometidos, no qual apresentaram uma taxa de $100 \%$ (cem por cento) de negatividade para o ácaro, também com uma regressão total do quadro clínico.

Como medida profilática, recomendou-se o uso de Fipronil Spray®, mensalmente, evitando novas infestações por ectoparasitas deste gênero.

\section{Resultados e Discussão}

Os resultados encontrados na avaliação física, como aspecto polvilhado, prurido e alopecia estão de acordo com as descrições feitas por Graig e Tell (1993), Mueller (2005) e Payane et al. (2005), que indicam que a principal região acometida 
pelo Lynxacarus radovskyi é o dorso. Alguns autores, como Jaffé et al. (2005) e Greve \& Gerrish (1981), citam como os membros posteriores sendo os mais acometidos. Porém, no presente trabalho a infestação ocorreu de forma generalizada, igualmente ao citado por Serra-Freire et al. (2002).

Os achados foram muito semelhantes aos encontrados por Figueiredo et al. (2004), onde 42 animais de um total de 613, que estavam com suspeita de esporotricose, foram diagnosticados com linxacariose. Em seu trabalho foi observado que os felinos machos eram três vezes mais parasitados, em relação as fêmeas. Contudo, Silva et al. (2021), relataram em seu trabalho o oposto. Fêmeas apresentaram 62,07\% de taxa de acometimento, enquanto que os machos tinham 21,96\%, num total de 29 animais positivados em sua pesquisa, onde não se observou influência do sexo, o que também se observa no presente trabalho.

O tratamento utilizado para os vinte e seis felinos do trabalho foi igual ao realizado por Aguiar et al. (2009) utilizando Fipronil Spray ${ }^{\circledR}$, semanalmente por 30 dias, onde foi obtido sucesso no tratamento do parasitismo. De forma muito parecida, Borba e Steuernagel (2009) também utilizaram Fipronil Spray®. Porém, o tratamento consistia em uma única aplicação nos cinco animais estudados, onde também obtiveram sucesso.

Existe ainda várias outras formas de tratamento, como através do uso da Ivermectina, com aplicação de duas doses, de $300 \mu \mathrm{g} / \mathrm{kg}$, por via subcutânea, com intervalo de duas semanas, como indicam Foley, (1991), Moriello (1994) e Medleau e Hnilica (2003). Outro método terapêutico eficaz, apontado por Silva et al. (2009), é a utilização da Selamectina, na formulação spot-on, com duas aplicações no intervalo de um mês. Souza et al. (2012) traz como outra forma de tratamento a associação de d-fenotrina e piriproxifeno para o controle do agente parasitário.

Com o presente estudo, foi possível concluir que os sinais clínicos característicos de linxacariose, sendo eles, lesões alopécicas multifocais, prurido generalizado, descamação e dermatite úmida e o aspecto polvilhado de "sal e pimenta", apresentados pelos animais acometidos, foram efetivamente tratados com o uso de Amitraz e Fipronil Spray®, em forma de banho e pulverização, todas as semanas, durante um mês.

\section{Conclusão}

A linxacariose é uma doença muito incomum de se encontrar no cotidiano da clínica de pequenos animais, mas ainda sim pode ser observado casos esporádicos desta enfermidade causada pelo Lynxacarus radovskyi. Mesmo os animais demonstrando apenas sinais corriqueiros de outras doenças fúngicas e dermatológicas como a presença de prurido e alopecia, bem como começar a demonstrar um visual de um animal muito sujo, a identificação do agente é essencial para o fechamento do diagnóstico e início rápido de um tratamento eficaz para a eliminação desse ectoparasita.

Sendo assim, os tricogramas de controle foram de suma importância para o fechamento do diagnóstico. A eficácia da terapia estabelecida à base de banhos com Amitraz e pulverização de Fipronil Spray® de forma semanal durante um mês foi comprovada, com os resultados apontando uma taxa de 100\% (cem por cento) de negatividade para a presença de ácaros do gênero Lynxacarus radovskyi, com os animais voltando a sua vida normalmente após o episódio.

\section{Referências}

Aguiar, J. et al. (2009). Infestação mista por Lynxacarus radovskyi e Felicola subrostratus em um gato na região de Porto Alegre, RS, Brasil, Acta Scientiae Veterinariae, 37(3): 301-305.

Borba, L. A. \& Steuernagel, E. (2009). Primeiro relato de cinco casos de linxacariose felina na cidade de Joinville, Santa Catarina, Brasil. MEDVET, Rev. Cient. Med. Vet, 7(20): 5-7.

Craig T. M. \& Tell P. D., Dubuisson L. M. \& Duibisson R. K. (1993). Lynxacarus radovskyi infestation in a cat. Journal of the American Veterinary Medical Association. 202: 613-614.

Faccini, J. L. H. \& Coutinho, V. (1986). Ocorrência de Lynxacarus radovski (Acari:Listrophoridae) em gatos domésticos no Brasil. Arquivos da Universidade Federal Rural do Rio de Janeiro, 9(1-2): 91-93. 
Faustino M. A. G., Messias J. B. \& Romeiro E. T. (2004). Infestação por Lynxacarus radoviskyi (Tenório 1974) em felinos - revisão. Clínica Veterinária. 53: $52-56$.

Greve, J. H. \& Gerrish, R. R. (1981). Fur mites (Lynxacarus) from cats in Florida. Feline Practice. 11(6): 28-30.

Heath A. C. G. \& Mariadas B. A. (1999). A New Zealand record for the cat fur-mite Lynxacarus (Felistrophorus) radovskyi Tenorio (Acarina: Astigmata: Listrophoridae). New Zealand Veterinary Journal. 47: 211-212.

Jaffé, E. et al. (2005). Infestação por Lynxacarus radovskyi em cães e gatos domésticos na cidade de Niterói (RJ): relato de caso. R. bras. Ci. Vet., 12(1-3): 110113.

Mueller R. S. (2005). Superficial mites in small animal dermatology. In: 50 Congresso Nazionale Multisala SCIVAC. Rimini, Itália. <http://www.ivis.org/proceedings/scivac/2005/Mueller4_en.pdf?LA=1>].

Oliveira, E. S. \& Vieira Neto, M. F. (2019). Linxacarose Felina: Revisão de Literatura, In: VI Encontro de Iniciação à Pesquisa, UNIFAMETRO 2019. Clínica e Biotecnologias aplicadas em Medicina Veterinária.

Payne P. A., Dryden M. W. \& Carter G. R. (2005). External Parasitic Diseases of Dogs and Cats. In: Carter G.R. \& Payne P.A. (Eds). A Concise Guide to Infectious and Parasitic Diseases of Dogs and Cats. International Veterinary Information Service. <http://www.ivis.org/signin.asp?url=/sp ecial_books/carter/carter7/chapter.asp?LA=1>]

Porto W. J. N. et al. (2002). Ocorrência do Lynxacarus radovskyi (Tenório, 1974) em gatos domésticos (Felis catus) na cidade de Maceió - AL. In: Congresso Brasileiro de Parasitologia Veterinária. (Rio de Janeiro, Brasil). 65 p.

Romeiro E. T. et al. (2007). Aspectos clínicos da infestação por Lynxacarus radovskyi em gatos procedentes da Região Metropolitana do Recife. Revista Clínica Veterinária. 69: 108-113.

Scott D. W. et al. (1996). Doenças parasitárias da pele. In: Muller and Kirk Dermatologia dos pequenos animais. (5a ed.), Manole, $374-376$ p.

Serra-Freire N. M. et al. (2002). Lynxacarus radovskyi - Diagnóstico e Tratamento em felinos de Belém, Pará. Revista Universidade Rural. Série Ciências da Vida, 22: 57-60.

Silva, G. P. et al. (2021). Distribuição e Diagnóstico de Lynxacarus radoviskyi em Gato Doméstico. Brazilian Journal of Development, 7(1): 47-54.

Silva, M. F. O. et al. (2009). Uso da Selamectina para o tratamento de dermatite felina por Lynxacarus radovsky: Relato de Caso. In: JORNADA DE ENSINO, PESQUISA E EXTENSÃO DA UFRPE, 9, Recife -PE. Anais... Recife-PE: UFRPE.

Souza, C. P. et al. (2012). Eficácia da associação de d-fenotrina e piriproxifeno no controle de Lynxacarus radovskyi em gatos domésticos. Revista Brasileira de Medicina Veterinária, 34 (1): 28-30.

Tenório J. M. (1974). A new species of Lynxacarus (Acarina: Astigmata: Listrophoridae) from Felis catus in the Hawaiian Islands. Journal Medical Entomology. 11: 599-604. 